\title{
Index rerum vol. 12
}

Absorption of d-xylose, 347 Aggressive behaviour and care, 49 Alzheimer's disease, 71, 129 Amylobarbitone as sedativum, 335 Anaemia, 339

Anaemia in elderly females, 18 Anaemia and Paget's disease, 93 Antibodies fixing complement, 186 Appendix, structure and iron content, 366 Amyloidosis and Alzheimer's disease, 129 Arteriosclerosis and complement fixing antibodies, 186 Arteriosclerosis and cyclandelate, 88 Arteriosclerosis and subarachnoid haemorrhage, 115 Arteriosclerosis and syncopal attacks, 229 Autoímmunity and Alzheimer's disease, 129

Barbiturates for sedation, 335

Beogex suppositories and constipation, 164

Beta/alpha lipoprotein index, 1

Bone, Paget's disease, 93, 241

Carbohydrate metabolism and hemiplegia, 99 Chromosomes and senile dementia, 71 Cerebral arteriosclerosis, 88, 115, 229

Cerebrovascular disease and cinnarizine, 297 Cerebrovascular insufficiency, 88 Cinnarizine in cerebrovascular disease, 297 Collagen and pituitary function, 65 Complement fixing antibodies, 186 Constipation, treatment of, 164 Cyclandelate and arteriosclerosis, 88

Dementia, senile, 71, 129

Dementia and disturbed behaviour, 49, 257,

267 Dementia and tranquillisers, 351 Dentine apposition, 360 Diazepam and muscle spasm, 168 Diploidicity and senile dementia, 71 Diverticulosis, jejunal, 80

Electrocardiography in the elderly, 193,

203, 321 Environment of nonagenarians, 175 Extrasystoles and electrocardiography, 193, 203,321

Fibel für die praktische Geriatrie (B), 377 Fibrillation and electrocardiography, 193

Geriatric care, changes in the demand, 257, 267

Geriatrie, Fibel (B), 377

Glucose tolerance test and beta/alpha lipoprotein index, 1

Glucose tolerance test and hemiplegia, 99

Growth hormone and pituitary function, 65

Haematological study of elderly females, 18 Haematology and vitamin K, 10 Haemoglobin content in blood, 339 Haemorrhage, subarachnoid, in the elderly,

115 Heart murmurs, 275

Hemiplegia and glucose tolerance test, 99 Heparin andtibodies, 186 Heredopathia atactica polyneuritiformis,

219

380

Index rerum

Herpes zoster and diaphragmatic paralysis, 
283 Hospital care and dementia, 49, 257, 267 Human aging and behaviour (B), 256

Hypopituitarism, 65

Immuno-cryourogenital treatment of prostate diseases, 302 Incontinence and care, 33, 288

Infections, venereal, 76 Iodine and pituitary function, 65 Iron content of the appendix, 366

Jejunal diverticulosis, 80

$\mathrm{K}$, vitamin deficiency, 10

Lipid disorders and complement fixing antibodies, 186 Lipids in the serum, 1 Lipoprotein in the serum, 1 Locomotion and care, 33, 235, 257, 267, 288

Malabsorption, 347

Malabsorption and osteomalacia, 80

Mental capability and care, 33, 40, 49, 149,

219 Mental function and cerebrovascular insufficiency, 88 Mental manifestations and

tachycardy, 203 Mobility and care, 33, 40, 257, 267, 288 Morbidity and anaemia, 18 Mortality

and hospital care, 40 Muscle spasm and diazepam, 168 Myocardial infarction and electrocardiography, 193 Myocardium, 193, 203, 275

Neoplastic diseases and complement fixing

antibodies, 186 Night care, need for, 33, 257, 267 Nonagenarians and environment, 175

Osteitis deformans, 93, 241 Osteomalacia and malabsorption, 80

Paget's disease and anaemia, 93, 241 Paralysis of the diaphragm and herpes

zoster, 283 Physical illness and care, 33, 40, 49, 149, 288 Pituitary function in the elderly, 65

Practolol in treatment of cardiac arrhythmias, 321 Prostate diseases, treatment of, 302

Refsum's disease, 219

Rehabilitation and geriatric care, 33, 49,

257, 267 Restlessness and tranquillisers, 351 Rickets and jejunal diverticulosis, 80 Rigidity and diazepam, 168

Sensory receptors in skin, 213

Serum, complement fixing antibodies, 186

Serum lipids and beta/alpha lipoprotein

index, 1 Skin and pricking pain threshold, 213 Skin thickness and pituitary function, 65 Social

club within a hospital, 235 Social vulnerability and care, 33, 40, 219,

235, 257, 267 Spasticity and diazepam, 168 Structure and iron content of the appendix,

366 Syncopal attacks, 229 Systolic murmurs, 275

Tachycardy, 193, 203, 321 Tooth, dentine apposition, 360 Tranquillisers and restlessness, 351

Veneral infections, 76 Vitamin K deficiency, 10

d-Xylose absorption, 347 